University of Wollongong

Research Online

Faculty of Informatics - Papers (Archive)

Faculty of Engineering and Information

Sciences

2005

\title{
Multi-channel FBG sensing system using a dense wavelength division demultiplexing module
}

\author{
Enbang Li \\ University of Wollongong, enbang@uow.edu.au \\ Jiangtao Xi \\ University of Wollongong, jiangtao@uow.edu.au \\ Yanguang Yu \\ University of Wollongong, yanguang@uow.edu.au \\ Joe F. Chicharo \\ University of Wollongong, chicharo@uow.edu.au \\ Jianquan Yao \\ Tianjin University
}

Follow this and additional works at: https://ro.uow.edu.au/infopapers

Part of the Physical Sciences and Mathematics Commons

\section{Recommended Citation}

Li, Enbang; Xi, Jiangtao; Yu, Yanguang; Chicharo, Joe F.; and Yao, Jianquan: Multi-channel FBG sensing system using a dense wavelength division demultiplexing module 2005.

https://ro.uow.edu.au/infopapers/2831

Research Online is the open access institutional repository for the University of Wollongong. For further information contact the UOW Library: research-pubs@uow.edu.au 


\title{
Multi-channel FBG sensing system using a dense wavelength division demultiplexing module
}

\author{
Abstract \\ Fiber Bragg grating (FBG) sensing is gaining attention in both scientific research areas and engineering \\ applications thanks to its distinguishing advantages including wavelength multiplexing capability, \\ miniature size, high sensitivity, immunity from electro-magnetic interference, etc. FBG sensing is based on \\ the detection of the shifted Bragg wavelength of the light reflected by a fiber grating which is sensitive to \\ various physical parameters such as strain and temperature. One of the challenging tasks in FBG sensing \\ is to determine the Bragg wavelength shift, which can be done by using an optical spectrum analyzer \\ (OSA). An OSA is suitable for laboratory tests, but not an ideal solution for field applications in term of \\ cost and convenience. Different wavelength demodulation methods have been developed for FBG sensing \\ purpose. One of them is employing a bulk linear edge filter to convert the wavelength shifts to intensity \\ variations. This method offers several obvious advantages including low cost and ability for dynamic \\ measurements. However, most of the edge-filter based FBG sensing systems are designed for single- \\ channel measurement. In this study, we propose and develop a multi-channel FBG sensing system based \\ on the edge-filtering technique. In order to demodulate multi-channel signal from FBG sensors, we \\ propose to use a dense wavelength division multiplexing (DWDM) module. The light signals coming from \\ wavelength-multiplexed FBG sensors are demultiplexed into individual channels and demodulated by the \\ pass-band edges. In the present study, a four-channel FBG sensing system has been demonstrated.

\section{Disciplines} \\ Physical Sciences and Mathematics

\section{Publication Details} \\ Li, E., Xi, J., Yu, Y., Chicharo, J. F. \& Yao, J. (2005). Multi-channel FBG sensing system using a dense \\ wavelength division demultiplexing module. In Y. Rao, O. Kwon \& G. Peng (Eds.), SPIE Advanced Sensor \\ Systems and Applications (pp. 211-218). USA: The Society of Photo-Optical Instrumentation Engineers.
}




\title{
Multi-channel FBG sensing system using a dense wavelength division demultiplexing module
}

\author{
Enbang $\mathrm{Li}^{\mathrm{a}}{ }^{\mathrm{b}}$, Jiangtao $\mathrm{Xi}^{\mathrm{b}}$, Yanguang $\mathrm{Yu}^{\mathrm{b}}$, Joe Chicharo ${ }^{\mathrm{b}}$ and Jianquan $\mathrm{Yao}^{\mathrm{a}}$ \\ ${ }^{a}$ College of Precision Instrument and Optoelectronics Engineering \\ Tianjin University, Tianjin 300072, P. R. China \\ ${ }^{\mathrm{b}} \mathrm{School}$ of Electrical, Computer and Telecommunications Engineering \\ Faculty of Informatics, University of Wollongong \\ Wollongong, NSW 2522, Australia
}

\begin{abstract}
Fiber Bragg grating (FBG) sensing is gaining attention in both scientific research areas and engineering applications thanks to its distinguishing advantages including wavelength multiplexing capability, miniature size, high sensitivity, immunity from electro-magnetic interference, etc. FBG sensing is based on the detection of the shifted Bragg wavelength of the light reflected by a fiber grating which is sensitive to various physical parameters such as strain and temperature. One of the challenging tasks in FBG sensing is to determine the Bragg wavelength shift, which can be done by using an optical spectrum analyzer (OSA). An OSA is suitable for laboratory tests, but not an ideal solution for field applications in term of cost and convenience. Different wavelength demodulation methods have been developed for FBG sensing purpose. One of them is employing a bulk linear edge filter to convert the wavelength shifts to intensity variations. This method offers several obvious advantages including low cost and ability for dynamic measurements. However, most of the edge-filter based FBG sensing systems are designed for single-channel measurement. In this study, we propose and develop a multi-channel FBG sensing system based on the edge-filtering technique. In order to demodulate multi-channel signal from FBG sensors, we propose to use a dense wavelength division multiplexing (DWDM) module. The light signals coming from wavelength-multiplexed FBG sensors are demultiplexed into individual channels and demodulated by the pass-band edges. In the present study, a four-channel FBG sensing system has been demonstrated.
\end{abstract}

Keywords: fiber Bragg grating, fiber sensing, wavelength division multiplexing

\section{INTRODUCTION}

A fiber Bragg grating (FBG) is formed by periodically modulating the refractive index of the core of an optical fiber. This all-fiber component can selectively reflect the light of a wavelength defined by the grating period and the refractive index of the fiber core. The potential applications of FBG devices in both optical fiber communications and fiber sensing areas had been realized immediately after its discovery in 1978 and, since then intensive research has been devoted to the development of FBG sensing systems and their applications ${ }^{1,2}$. FBG sensing provides unique advantages over conventional electrical sensors and can be used in applications where electrical sensors are limited. These advantages include:

1. High bandwidth and multiplexing capabilities;

2. Immunity to electromagnetic and RF interference;

2. Immunity to chemicals, radioactivity, corrosion and lightning;

3. Intrinsic sensing of multiple measurands without referencing;

4. Stability, repeatability and durability against harsh environments; and

6. High sensitivity and resolution, and fast response.

In recent years, FBG sensing is gaining considerable attention in both scientific research areas and engineering applications. This is evidenced by the exponentially increased numbers of published research works in this area. FBG sensing has been widely used to measure strain, temperature, and pressure in various fields ranging from the 
so-called smart structures, structural health monitoring to aerospace industries. FBG sensing and its application in various situations have been well described in a number of recently published reviews ${ }^{3-5}$.

FBG sensing is based on the detection of the shifted Bragg wavelength of the light reflected by a fiber grating which is sensitive to strain and temperature. One of the important and challenging tasks in FBG sensing is to accurately determine the Bragg wavelength shift caused by the measurands. Optical spectrum analyzers (OSAs) are frequently used to measure the wavelength shifts in FBG sensing systems. An OSA is most suitable for laboratory tests, but not an ideal solution for field applications in terms of the cost and convenience. Different wavelength demodulation methods have been developed for FBG sensing purpose, including scanning Fabry-Perot filter-based interrogation, tunable acousto-optic filter interrogation, and prism/CCD-array technique ${ }^{2,5}$. Another method is employing a bulk linear edge filter to convert the wavelength shifts to intensity variations ${ }^{6,7}$. This method offers several obvious advantages such as low cost and ability for dynamic measurements. However, most of the edge-filter based FBG sensing systems are designed for single-channel measurement. As mentioned above, one of the most distinguishing features of FBG sensing is its wavelength multiplexing ability, which is extremely important for constructing multipoint sensing systems and networks. On the other hand, multi-point sensing systems with fast response are highly desirable for a number of applications such as arrayed hydrophone systems.

In this study, we further explore the edge-filtering technique to multi-point FBG sensing. In order to demodulate multi-channel signal from multiple FBG sensors, we propose to use a dense wavelength division multiplexing (DWDM) module. We demonstrate that by using a DWDM module, channel separation and wavelength demodulation can be achieved simultaneously, leading to a significantly simplified sensing system and a reduction of the cost for constructing the system. In the following sections, we first introduce the principle and the structure of the proposed sensing system, and then we present a theoretical model to describe the system. In Section 4, experiments and results are given. We conclude in Section 5.

\section{PRINCIPLE OF OPERATION}

Wavelength division multiplexing has been widely used in optical communications systems to increase the capacity of information carried by a single transmission fiber. This concept is also applicable to fiber sensing in general ${ }^{8}$, and FBG sensing in particular, ${ }^{9} 10$. Many WDM or DWMD components and modules developed for optical communications purposes can be directly used for sensing as well. The aim of this study is to develop a low-cost multi-channel FBG sensing system by taking the advantage of the optical fiber components and modules commercially available for optical communications systems.

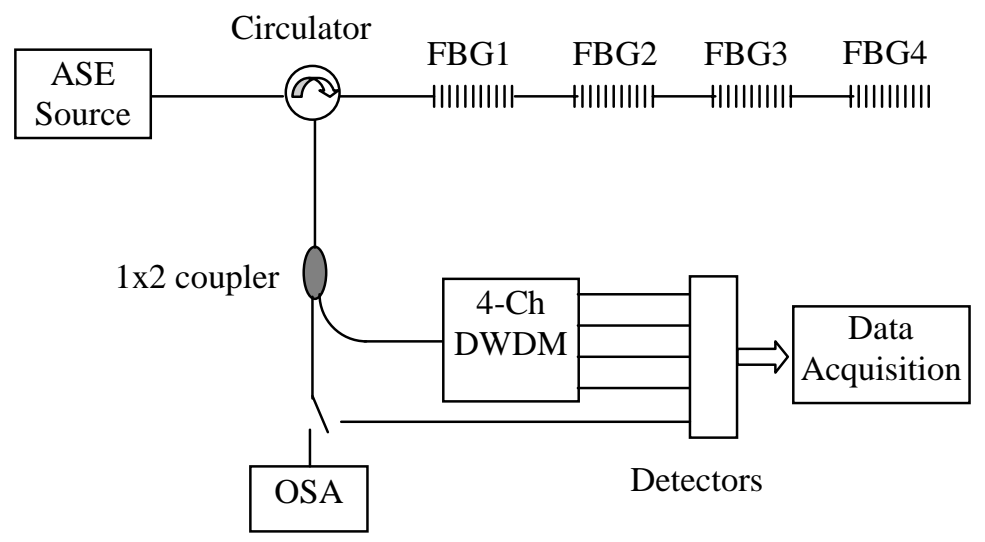

Fig.1 Schematic diagram of a four-channel FBG sensing system developed

A DWDM demultiplexing module is designed for separating an optical signal containing multiple wavelengths into individual channels. It consists of a series of bandpass filters using thin film, FBG, or arrayed waveguide technologies. Depending on the different filtering requirements, the filters have different spectral shapes. Similar to 
the bulk filters, the edges of the DWDM filters can be used as edge filters to demodulate the wavelength shifts in FBG sensing. In this case, both wavelength demultiplexing and wavelength demodulating in a multiple FBG sensing system can be simultaneously realized. By properly selecting a DWDM module and designing sensing FBGs to locate the wavelength of an individual FBG at the edge of the related channel, a low-cost multi-channel sensing system which is suitable for dynamic measurement can be developed.

\section{SYSTEM DESCRIPTION}

Shown in Fig. 1 is a schematic diagram of a four-channel FBG sensing system we developed. The key part of the system is a four-channel DWDM module (manufactured by Oplink). The module is a thin film filter-based device, containing four channels. In contrast to the AWG or FBG-based devices, thin film filters have several advantages including smooth spectral response and thermal stability, which are critical for an edge filter-based FBG wavelength demodulation scheme. The central wavelengths of the bandpass filters are $1552.52 \mathrm{~nm}, 1554.94 \mathrm{~nm}, 1557.36 \mathrm{~nm}$ and $1559.79 \mathrm{~nm}$ respectively. Figure 2 shows the measured transmission spectra of the demultiplexing module. With a channel spacing of $2.4 \mathrm{~nm}$, or $300 \mathrm{GHz}$, an isolation better than $30 \mathrm{~dB}$ can be achieved between two adjacent channels. The maximum insertion loss from the input port to individual channels is less than $1 \mathrm{~dB}$.

The wavelengths of the sensor gratings are selected according to the wavelengths of the DWDM module. In principle, we can use either the rising edge or the falling edge of a filter channel as the wavelength demodulator once the FBG wavelength is properly located at the edge of a filter. It is also possible to use rising edges of some filters and falling edges of the others, depending on the availability of the FBG sensors, which provides further flexibility in constructing a multi-point sensing system.

In order to demonstrate the proposed scheme, we selected four FBGs with their central wavelengths less than the relevant filter channels. The FBGs can be tuned to make their central wavelengths locate at the positions as we intend. The reflection spectra of the FBGs cascaded in a signal fiber are shown in Fig.3. The spectra were measured by an OSA at room temperature $\left(25^{\circ} \mathrm{C}\right)$ without tension applied.

Referring to Fig.1, light from a broadband source (amplified spontaneous emission from an erbium-doped fiber amplifier) is transmitted to the sensor FBGs through a three-port optical circulator. The light signals reflected by the wavelength-multiplexed FBG sensors are directed to a 1x2 coupler by the circulator. One of the outputs of the coupler is used to monitor the spectra of the reflected optical signals and to measure the optical power of the signals. The power measurement will be used in the calculation of the wavelength shifts, which will ensure that the possible power variations of the broadband light source and the loss of the transmission fiber are taken into account.

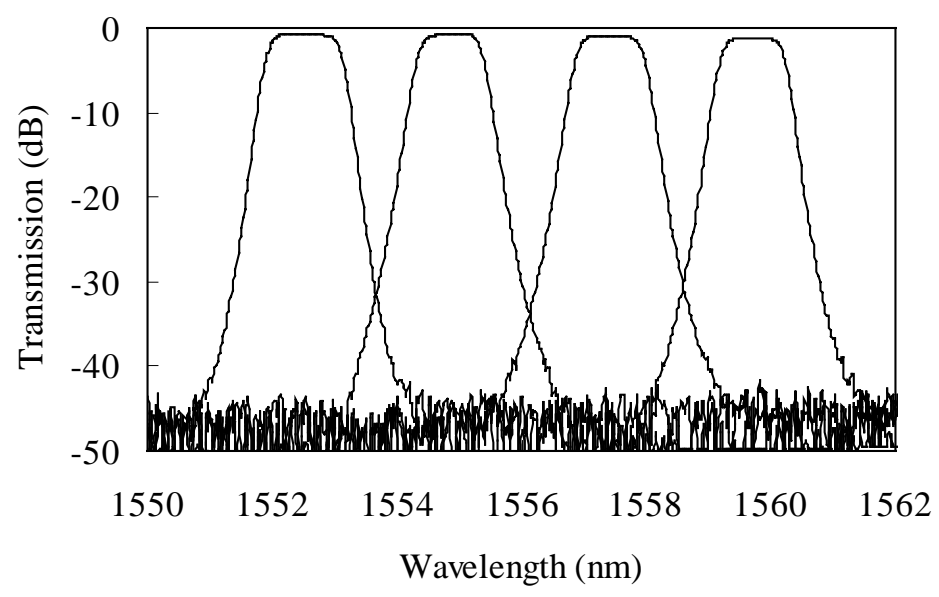

Fig.2 Measured transmission spectra of the DWDM module 
The second port of the $1 \times 2$ coupler is connected to the input port of the DWDM module, where the back-reflected signals from sensor FBGs are demultiplexed and demodulated. The output signals of the DWDM module together with the reference signal are detected by individual photodetectors. The photocurrent signals are converted to voltages, then amplified before connecting to a multi-channel data acquisition card plugged in a PC computer where the data are stored and wavelength shifts are calculated.

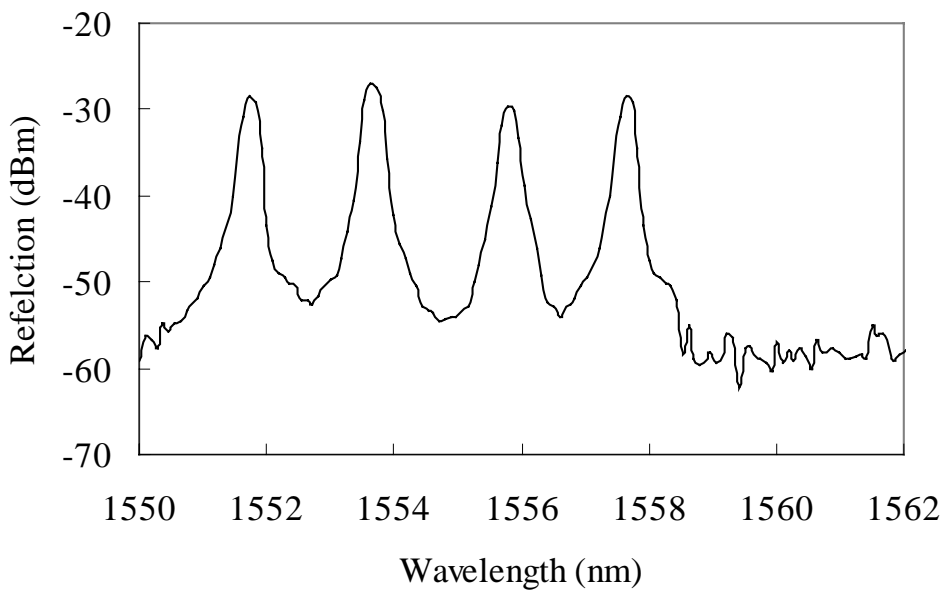

Fig.3 Measured reflection spectra of four sensor FBGs

\section{ANSLYSIS AND SIMULATION}

In this section, we present an analysis of the demodulation process and simulate it with the known parameters. As mentioned before, the DWDM module can provide a better than $30 \mathrm{~dB}$ isolation between two adjacent channels. Under this condition, we can assume that the channel cross-talk is negligible, and hence we treat every channel independently without considering other channels. The simulation presented below is for the first channel, however, the same method can be applied to other channels.

From the coupled mode theory, the reflectance of an FBG can be expressed as ${ }^{11}$

$$
R(L, \lambda)=\frac{\Omega^{2} \sinh ^{2}(S L)}{\Delta \beta^{2} \sinh ^{2}(S L)+S^{2} \cosh ^{2}(S L)},
$$

where, $\lambda$ is the wavelength; $\mathrm{L}$ is the length of the grating; $\Omega$ is the coupling coefficient; $\Delta \beta=\beta-(\pi / \Lambda) ; \beta$ is the eigen propagation constant; $\Lambda$ is the grating period; and $S=\left(\Omega^{2}-\Delta \beta^{2}\right)^{1 / 2}$.

From Eq.(1) the reflectance of a grating can be calculated if the grating parameters are known. From the spectral shapes of the filters as shown in Fig.1, we can assume that the transmission spectra of the filters have a superGaussian shape, that is

$$
T(\lambda)=\alpha_{f} \exp \left[-\left(\frac{\left|\lambda-\lambda_{i}\right|}{B_{i}}\right)^{n}\right]
$$

where, $\alpha_{f}$ is a constant representing the insertion loss introduced by the DWDM module; $\lambda_{i}$ and $B_{i}$ are the central wavelength and bandwidth of the filter channel considered; and $\mathrm{n}$ is the order of the super-Gaussian function. Through calculations, we found that when $n=6$, Eq.(2) gives an excellent curve-fit to the spectra of the filters.

The power of the signal reaching the photo-detector can be expressed as 
$P=\alpha \int_{\lambda_{1}}^{\lambda_{2}} R(L, \lambda) T(\lambda) d \lambda$,

where, $\alpha$ is a constant, and $\left(\lambda_{1}, \lambda_{2}\right)$ is the bandwidth of the broadband source used in the system.

We simulated the first channel by using Eqs(1)-(3) and the following grating parameters: $\mathrm{L}=10 \mathrm{~mm}$; the effective refractive index, $n_{\text {eff }}=1.46$; and the amplitude of the refractive modulation, $\Delta n=0.0001$. The calculated optical power as a function of wavelength is shown in Fig.4. Note that the power is normalized by its maximum value. Also shown in Fig.4 are the measured data. It can be seen that excellent agreement exists between the calculated powers and the measured results.

\section{EXPERIMENTS AND RESULTS}

In order to demonstrate the proposed sensing system and also characterize the individual channels, we carried out two series of tests. The first one is to test the performance of the system with static strains, and the second is focusing on the dynamic measurements.

An experimental set-up was built according to the diagram shown in Fig.1. An ASE with an output power of about 5 $\mathrm{mw}$ with a band ranging from $1526 \mathrm{~nm}$ to $1566 \mathrm{~nm}$ was used as the broadband light source. An optical spectrum analyzer (ADVANTEST Q8347) was also employed to monitor the spectra of the signals reflected by the sensor FBGs and to measure the wavelength shifts. An electronic unit with four photo-detectors and pre-amplifiers was designed for detecting the light signals from the DWDM outputs. We also used a dual-channel power meter (Newport 2832C) to measure the optical power in the experiments.

\subsection{Static measurements}

In order to test the performance of each channel, we tuned the wavelengths of the FBGs to the rising edges of their corresponding filters by mechanically stretching the gratings with specially designed holders. The power of each channel was measured by the power meter, and the wavelength was measured by the OSA. The results for the first channel is given in Fig.4, which indicates that when the grating is stretched, the wavelength of the signal reflected by the grating increases and the optical power also correspondingly increases. The linear range of the edge filter is about $0.35 \mathrm{~nm}$ for all the four channels. With the current set-up, a resolution better than $1 \mathrm{pm}$ can be achieved for each channel.

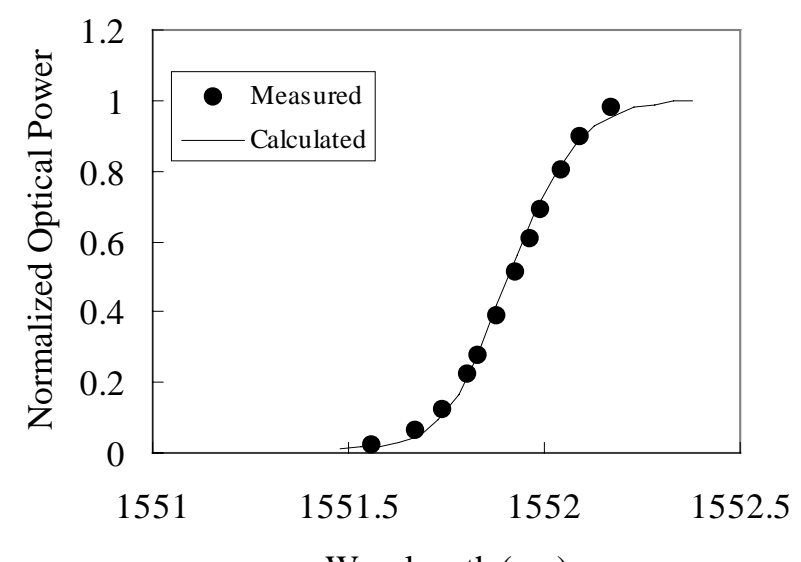

Wavelength (nm)

Fig. 4 Calculated and measured response of the first channel

In a separate test, we tuned a grating to locate its wavelength at the falling edge of the fourth channel, and recorded the Bragg wavelength and the power of the reflected signal. The test results show that the falling edge has a similar 
slop (opposite sign) and resolution. This demonstrates that both the rising and falling edges of a filter can be utilized for wavelength demodulation purposes.

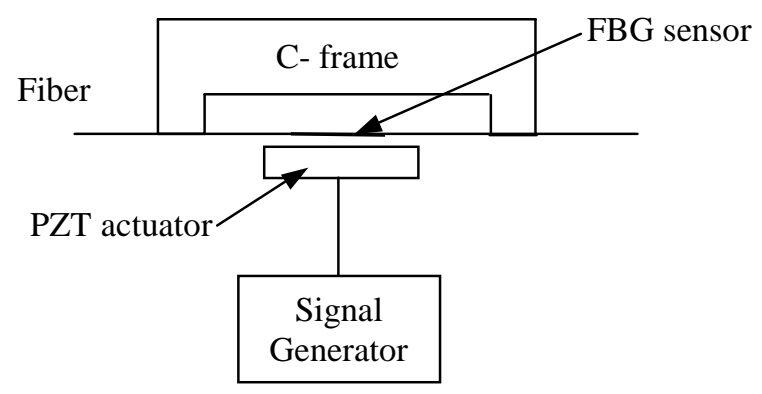

Fig.5 Experimental set-up for vibration measurements
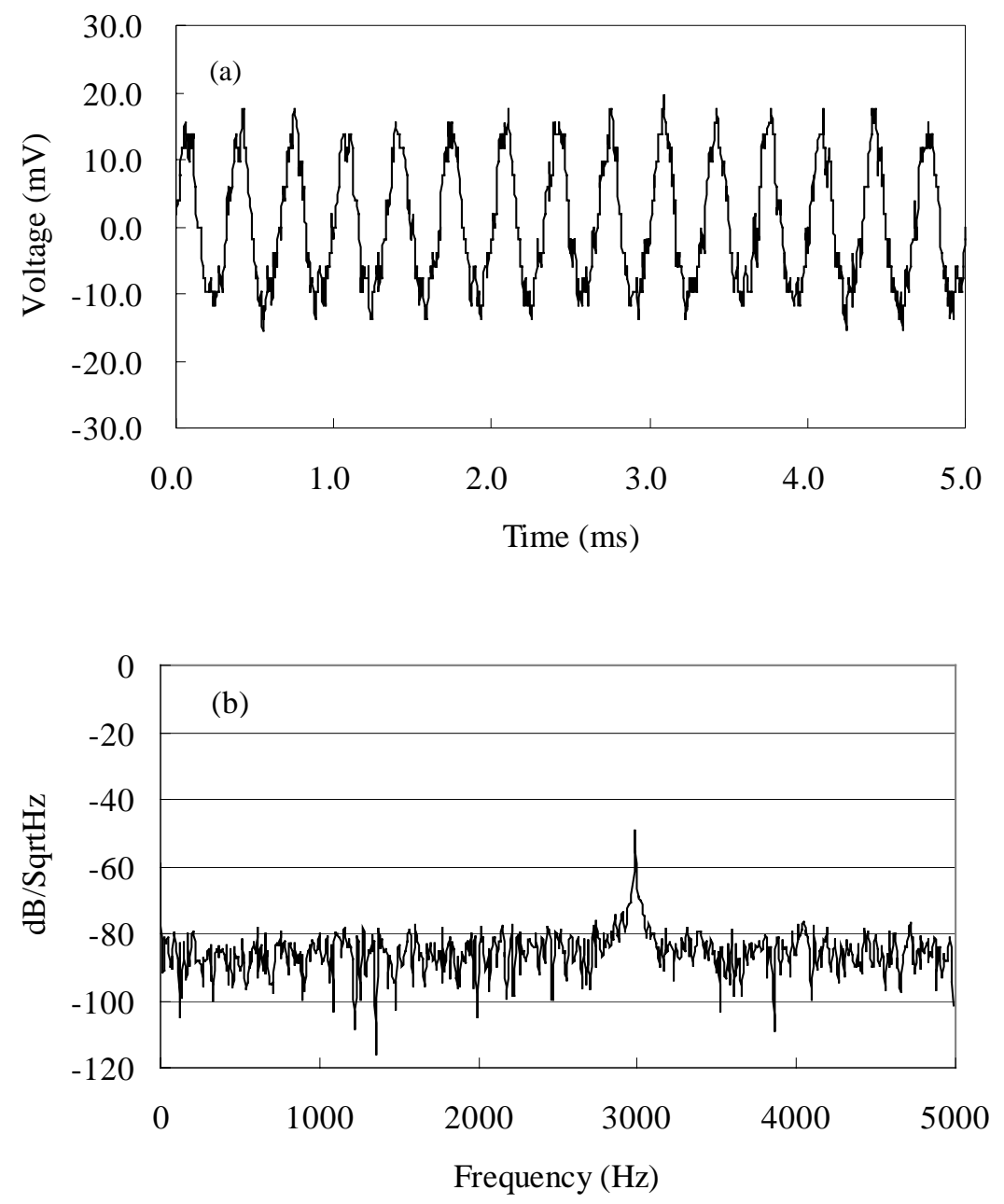

Fig.6 Recorded vibration signal (a) and its spectrum (b) 


\subsection{Dynamic measurements}

In order to test the dynamic performance of the sensing system, we measured the vibration generated by a PZT actuator. Show in Fig.5 is a diagram of the set-up. The sensor FBG was fixed to a C-frame with a tension to ensure that its wavelength was at the rising edge of the second channel. The PZT actuator, which was driven by a sinusoidal voltage signal generated by a function generator, was attached to the sensor FBG. The output of the second channel was connected to the power meter, and the output of the power meter was connected to an input of a digital oscilloscope board (NI 5102) plugged in a PC computer, For comparison purpose, the driving signal was also monitored by using the second channel of the NI 5102 board. Both the driving signal and the detected vibration signal were recorded and analyzed by using VirtualBench software (from NI).

Shown in Fig. 6 are the measured vibration signal and its spectrum when the PZT driving signal was tuned to about 3 kHz. Fig. 7 shows the waveform and spectrum of the driving signal.
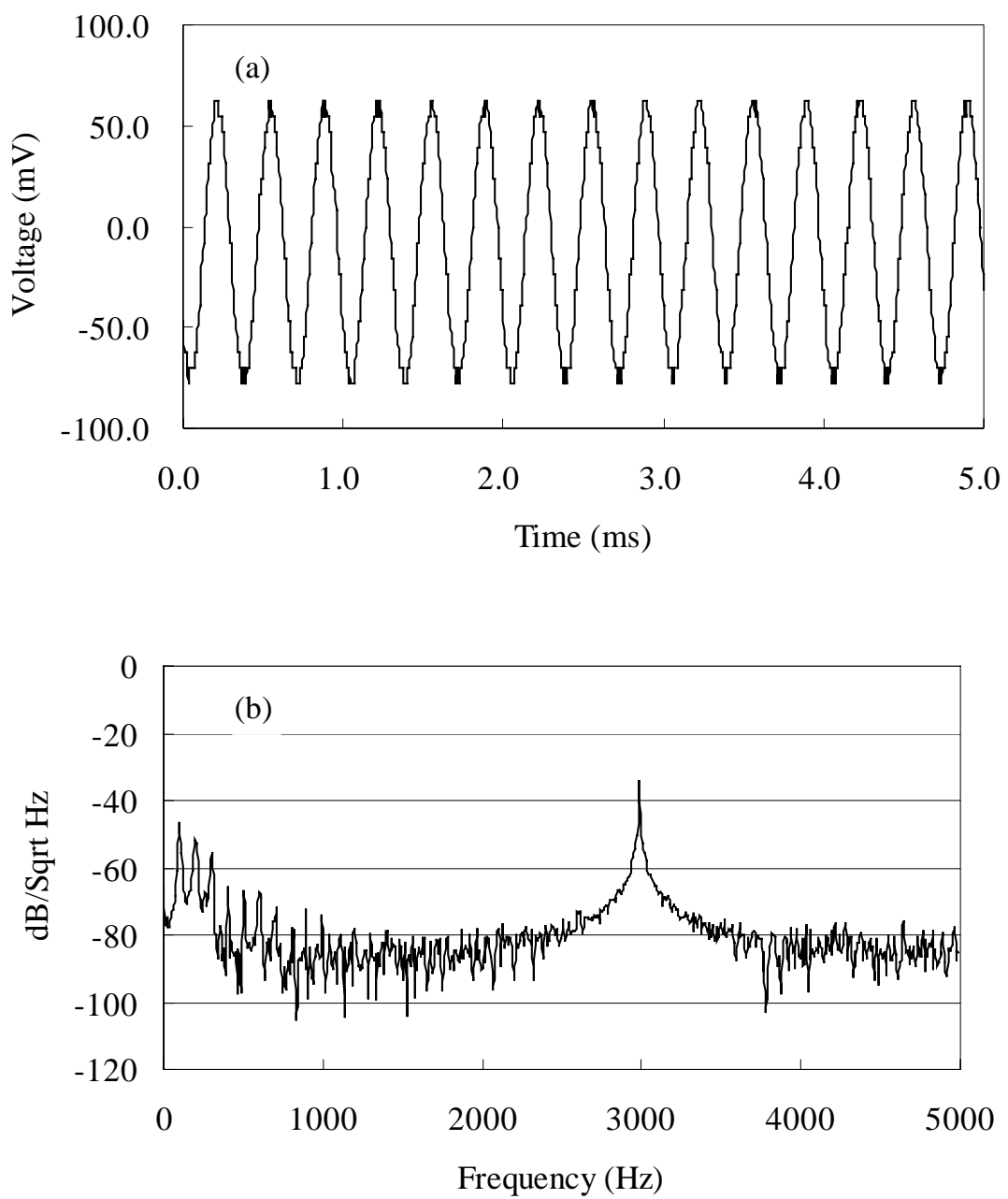

Fig.7 PZT driving signal (a) and its spectrum (b)

\subsection{Channel cross-talk}

The possible cross-talk in a multi-point sensing system using wavelength-multiplexing should be eliminated or minimized. The worst case cross-talk normally happens between two adjacent channels. In order to identify the possible cross-talk, we used the set-up shown in Fig.5 to generate vibration signals in the second channel and monitored the outputs from the first and third channels. The experiments have shown that there are no detectable 
signals from the two adjacent channels. This verifies that the $30 \mathrm{~dB}$ isolation between any adjacent channels is high enough to eliminate channel cross-talk for our sensing purpose.

\section{CONCLUSIONS}

We have proposed and developed a multi-point FBG sensing system by using a commercially available DWDM module. The functions of the DWDM module are two-fold: (1) it effectively separates the wavelength-multiplexed optical signal to individual channels; and (2) the edges of the channel filters act as wavelength demodulators which convert the wavelength shifts caused by the measurands to optical power variations. We have demonstrated a fourchannel system and applied it to vibration measurements. It should be noted that the channel number can be easily scaled up by selecting a DWDM module with more channels. The system is most suitable for measuring dynamic processes, such as vibrations and dynamic strains. As the prices of optical communications components are continuously dropping, the method proposed in this study offers a significant advantage in terms of the cost for constructing an FBG sensing system with multiple sensors.

\section{REFERENCES}

1. R. Kashyap, Fiber Bragg Gratings, Academic Press, San Diego, 1998, pp. 1-10.

2. A. Othonos and K. Kalli, Fiber Bragg Gratings: Fundamentals and Applications in Telecommunications and Sensing, Artech House, Boston, 1999, pp. 1-6.

3. A. D. Kersey, M. A. Davis, H. J. Patrick, M. LeBlanc, K. P. Koo, C. D. Askins, M. A. Putnam and E. J. Friebele, "Fiber grating sensors," Journal of Lightwave Technology, Vol. 15, 1442-1463, 1997.

4. Y. J. Yao, "Recent progress in applications of in-fiber Bragg grating sensors," Optics and Lasers in Engineering, Vol. 31, 297-324, 1999.

5. Y. Zhao and Y. Liao, "Discrimination methods and demodulation techniques for fiber Bragg grating sensors," Optics and Lasers in Engineering, Vol. 41, 1-18, 2004.

6. S. M. Melle, K. Liu and R. M. Measures, "A passive wavelength demodulation system for guided-wave Bragg grating sensors,” IEEE Photonics Technology Letters, Vol. 4, 516-518, 1992.

7. M. A. Davis and A. D. Kersey, "All-fiber Bragg grating strain-sensor demodulation technique using a wavelength division coupler," Electronics Letters, Vol. 30, 75-77, 1994.

8. S. P. Christmas, D. A. Jackson, P. J. Henderson, L. Zhang, I. Bennion, T. Dalton, P. Butler, M. Whelan and R. Kenny," Measurement Science and Technology, Vol. 12, 901-905, 2001.

9. A. Fernandez, F. Berghmans, B. Brichard, P. Megret, M Decreton, M. Blondel and A. Delchambre, "Multicomponent force sensor based on multiplexed fibre Bragg grating strain sensors," Measurement Science and Technology, Vol. 12, 810-813, 2001.

10. L. Zhang, Y. Liu, J. A. R. Williams and I. Bennion, "Enhanced FBG strain sensing multiplexing capacity using combination of intensity and wavelength dual-coding technique," IEEE Photonics Technology Letters, Vol. 11, 1638-1640, 1999.

11. D. K. W. Lam and B. K. Garside, "characterization of single-mode optical fiber filters," Applied Optics, Vol. 20, 440-445, 1981. 